\title{
Ultra-high precision CMMs and their associated tactile or/and optical scanning probes
}

\author{
H. Nouira ${ }^{1, \star}$, R.H. Bergmans ${ }^{2}$, A. Küng ${ }^{3}$, H. Piree ${ }^{4}$, R. Henselmans ${ }^{5}$, and H.A.M. Spaan ${ }^{6}$ \\ 1 Laboratoire Commun de Métrologie (LCM), LNE, 1 Rue Gaston Boissier, 75015 Paris, France \\ 2 VSL Dutch Metrology Institute, Thijsseweg 11, 2629JA Delft, The Netherlands \\ 3 Federal Institute of Metrology (METAS), Lindenweg 50, 3003 Bern-Wabern, Switzerland \\ 4 SMD, Federal Public Service Economy - Metrology - National Standards, Koning Albert II laan 16, 1000 Brussel, Belgium \\ 5 TNO, Opto-mechatronics Department, Stieltjesweg 1, 2628 CK Delft, The Netherlands \\ ${ }^{6}$ IBS Precision Engineering bv, Bedrijfsweg 1, 5683 CM Best, The Netherlands
}

Received: 25 February 2014 / Accepted: 28 April 2014

\begin{abstract}
Optical and tactile single scanning probes usually are used in dimensional metrology applications, especially for roughness, form, thickness and surface profile measurements. To perform assessments with nanometre level of accuracy, specific ultra-high precision machines have been developed by the National Metrology Institutes (NMIs) such as the LNE, VSL, METAS, SMD, generally in collaboration with industrials and academics partners. Such developments are not devoted only to NMIs but many industrials develop and commercialize their own ultra-high precision machines as the IBSPE and TNO companies. All these machines provide optical and tactile precise measurements and cover a large domain of application such as the form's characterization of optical lenses. In this paper the performance and capability of ultra-high precision machines of some National Metrology Institutes (LNE, VSL, SMD and METAS) and industrials companies (TNO and IBSPE), involving together in the IND10 European EMRP project titled "Optical and tactile metrology for absolute form characterization", will be detailed. Theirs probing systems and accuracies levels will be evoked. Relevant results especially for measuring optical lenses will be also presented and discussed.
\end{abstract}

Keywords: Measuring machine, chromatic confocal probe, tactile probe, error sources, dimensional and mechanical metrology, evaluation

\section{Introduction}

Measuring optical surfaces with an uncertainty less than few nanometres for aspherical lens remains a real challenge in industry. Thus, under the European Metrology Research Program a three year project started since 2011 to improve the measurement of high quality optical surfaces of lenses and mirrors. It encompasses a multitude of National Metrology Institutes (NMIs) and other stakeholders.

In the field of ultra-precision 3D metrology, various small-volume coordinate measuring machines (CMMs) have been developed [1-4]. These machines typically feature 3 D measuring ranges less than $100 \times 100 \times 100 \mathrm{~mm}^{3}$, and usually they apply the same set of fundamental principles.

The main one consists in achieving high positioning and measuring accuracy with perfect respect to Abbe principle [5]. To comply with the Abbe principle, a

\footnotetext{
${ }^{\star}$ Correspondence: hichem.nouira@lne.fr
}

measuring system should be aligned with the distance to be measured.

The precision of such machines usually relay on the quality of the mechanical guiding elements, which usually consists of ultra-high precision air-bearing guide [4]. All these machines also apply the dissociated metrological structure principle $[6,7]$ where the metrology frame is decoupled from the rest of the machine and remains stress free to properly perform its own function consisting in tracking the $X$-Y-motion of a table with respect to the Abbe principle by using laser interferometers of sub-nanometre resolution. The selection of zerodur materials for the table allows designing non-symetric CMM machines since the thermal dilatation of such material is negligible, especially when the environment temperature is well controlled in a range of $\pm 0.2{ }^{\circ} \mathrm{C}$.

Both systematic and random errors motions of the ultra-high precision guiding elements are of few tenth of nanometres. For this reason the laser interferometers allow only to improve the knowledge of the coordinates of the zerodur table supporting the workpiece to the nanometre level of accuracy. 
The performance of CMMs depends on the metrology frame stability and the performance of the single scanning probe. Two type of probes can be used: optical and tactile probes. Usually tactile probes allow more accurate measurement then optical probes [8]. However, with technology progress, some industrial companies and NMIs succeeded developing new optical probes able to ensure measurements at the nanometre level of accuracy with high acquisition frequency (very short time) $[9,10]$.

In this paper LNE, VSL, SMD, METAS, TNO and IBSPE ultra-high precision coordinate measuring machines will be presented and discussed in particular scope of optical lenses metrology. The tactile and optical scanning probes which equip those machines will be specially detailed.

\section{LNE measuring apparatus}

The apparatus performs three independent motions $(x$, $y$ and $z$ ) generated by three independent high-precision mechanical guiding systems equipped with encoder. The horizontal ( $x$ and $y$ ) guiding systems support the table made in zerodur material, and is able to move in dynamic or static operation. The zerodur table is fixed on the guiding system with isostatic link. The vertical guiding system moves the structure supporting the measuring probe along the $z$-axis. Each $x$ and $y$ motion is controlled by a laser interferometer in order to locate the target with nanometre level of accuracy. The $z$-motion is controlled by a differential laser interferometer to shorten the metrology loop and to guarantee a similar level of accuracy. The supporting structure is made in massive granite materials and supports all the guiding systems. The metrology frame, represented in Figure 1, is made with invar material to be less sensitive to the environment and structure thermal deviation. Any vertical variation of the main granite supporting structure due to both thermal drift and its own masse only slightly influences the metrological structure by a few nanometres since this variation is indirectly compensated by the differential laser interferometer. The configuration of the machine allows measurements of the target with optical and tactile single point scanning probes.

The architecture of the machine perfectly fulfils the Abbe principle along all three axes. The measuring single scanning probe is collinear to the $z$-differential laser beam (1) and represents the "measuring Abbe $z$-axis". The horizontal $x$ and $y$-laser interferometers beams and the tactile probe or the focus point for the optical confocal probe are designed to be in the same $X Y$-plan and axis (Abbe $x$-axis and Abbe $y$-axis). These Abbe axes never change because the measuring probe and the $x$ and $y$-laser interferometers are fixed on the same invar metrological structure. Any vertical motion of the metrological structure induces a similar vertical motion of the $x$ and $y$-laser interferometers and the measuring single scanning probe. The $z$-laser interferometer is fixed on the supporting structure. Each laser interferometer is fixed to an intermediary positioning mechanism (two translations and one rotation) in order to

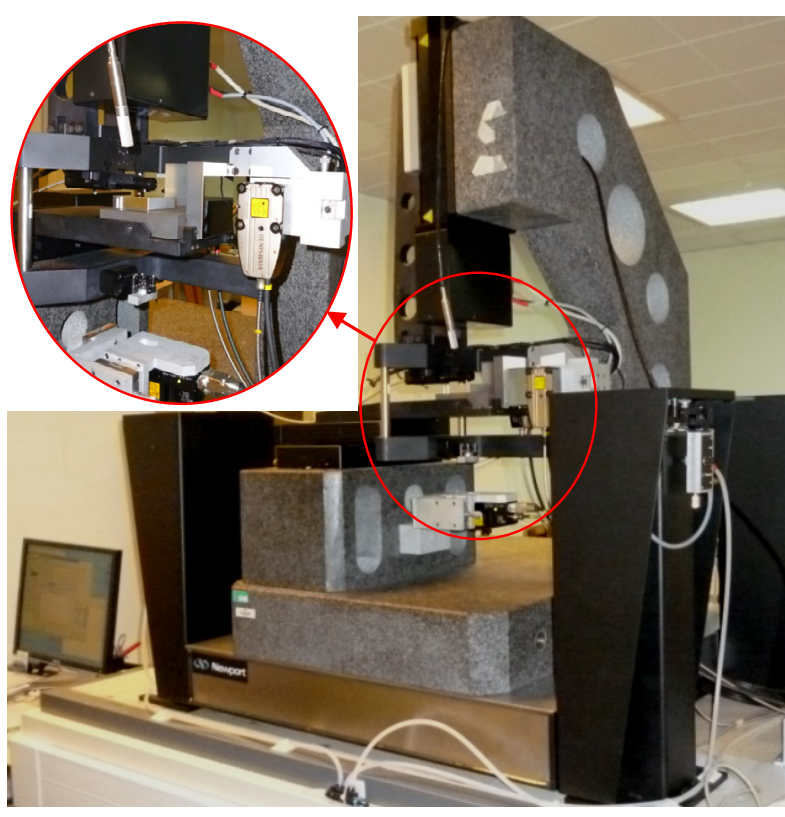

Fig. 1. LNE ultra high precision measuring machine.

adjust the alignment of the laser beam to each respective mirror [8].

The entire $x$ and $y$-working range is equal to $50 \mathrm{~mm}$. The $z$-working range can reach $100 \mathrm{~mm}$ and depends on the working range of the measuring probe. In-situ calibration of the measuring probe can be achieved by moving the metrological structure which contains the optical/tactile probe along the $z$-axis fulfilling the Abbe principle. The measuring probe is adjusted to be collinear to the differential laser beam (2) and represents the "calibration Abbe $z$-axis". For this operation, the zerodur table is kept unchanged and only the reference mirror and metrological structure are moved. The calibration metrology loop slightly differs from the measuring metrology loop. The recorded measuring probe data are then compared to the $z$-laser interferometer.

In order to establish an uncertainty budget, the motion errors of the mechanical guiding systems, the Abbe errors, the cosine errors, the dynamic operation of the machine, the geometry of the zerodur table, the thermal drift and the probe errors were considered and evaluated. The LNE's apparatus has the capability of calibrating artefacts with an accuracy of less than $30 \mathrm{~nm}$ when the travel range of the tactile probe does not exceed $150 \mu \mathrm{m}$. However, the value of the uncertainty strongly depends on the behaviour of the measuring probe.

\section{Confocal probe, inductive probe}

Usually, a high percent of the uncertainty is related to the behaviour of the measuring optical or tactile probe. To reduce this effect, the behaviour of the measuring probe is characterized under similar environment conditions, by using an external test bench. 


\subsection{Optical confocal chromatic probe}

The working principle of confocal chromatic probe for surface profiling is reported in $[9,10]$. We usually deal with a white light source (LED), where the spectral components are focused at different distances along the optical axis. Only the wavelength that is best focused on the surface of the object passes through a mechanical filter called the pinhole. The reflected light is collected and analyzed with a spectrometer (diffraction grating).

\subsection{Inductive probe}

Inductive probes are based on the variation of a magnetic field induced by a primary coil and sensed in a secondary sense coil. Thus a change in position of an iron core in the coils builds a linear variable differential transformer, which modulates the amplitude and phase of the voltage measured in the sense coil. Such probes present a very high dynamic range and an often-rugged construction. Due to their mechanical nature, they have lower repeatability, as coils do not always return to the same position between readings. However, the bias errors of these probes are very low: only a few nanometres.

The used inductive probe has a stylus tip angle of $90^{\circ}$, a tip radius of $2 \mu \mathrm{m}$ and a static measuring force of less than $0.7 \mathrm{mN}$. It can be used over 3 ranges of measurements: 0-100 $\mu \mathrm{m}$ (smaller range), 0-500 $\mu \mathrm{m}$ (medium range) and $0-1000 \mu \mathrm{m}$ (larger range).

\subsection{Investigation of the behaviour of both confocal chromatic and inductive probes}

The behaviour of the confocal and inductive probe is investigated in-situ by comparing it to information given by the differential laser interferometer. Since the confocal probe will be used in dynamic mode, a speed test is performed on the profilometer between 10 and $100 \mu \mathrm{m} / \mathrm{s}$. The recorded signal is modelled by 2040 partial linear models covering the whole travel range of the probe and the estimated linear residuals vary within $\pm 14 \mathrm{~nm}$. The standard deviation of the residuals is equals to $\pm 1.5 \mathrm{~nm}$ and centred on $y$-coordinate $=0$ (Fig. 2a). This choice leads to processing a big number of linearization in real time. In addition, when increasing the speed of the confocal probe's motion, under calibration, the residuals are unchanged, even though, the number of data decreases as the speed increases.

The behaviour of the inductive probe is investigated in-situ at first for the smaller working range. The recorded data are modelled by an orthogonal ninth order polynomial fitting curve. The nonlinear residuals are presented in Figure $2 \mathrm{~b}$ and vary within $\pm 20 \mathrm{~nm}$. The standard deviation varies between $\pm 6.5 \mathrm{~nm}$. The repeatability of the inductive probe has been tested and is better than $3 \mathrm{~nm}$.

A similar test is performed over the medium range and the results are presented in Figure 2c. The nonlinear residuals vary within $\pm 50 \mathrm{~nm}$ and the standard deviation varies

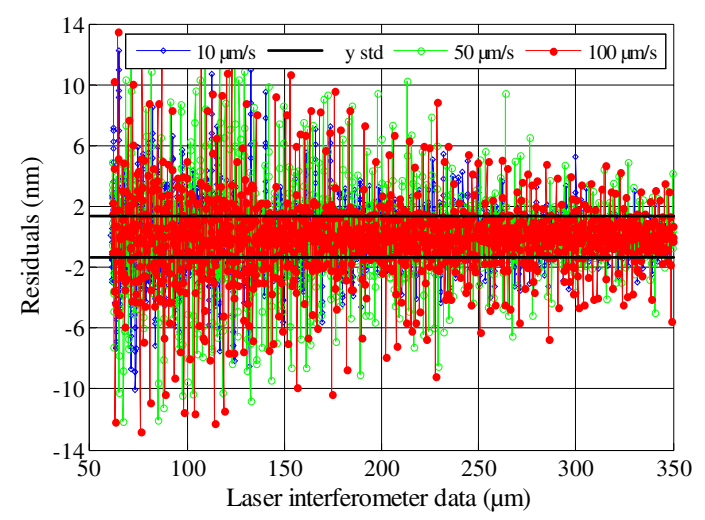

(a)

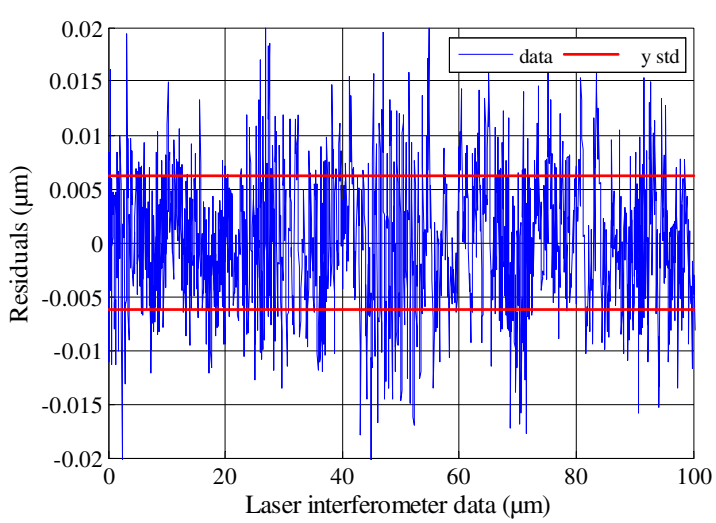

(b)

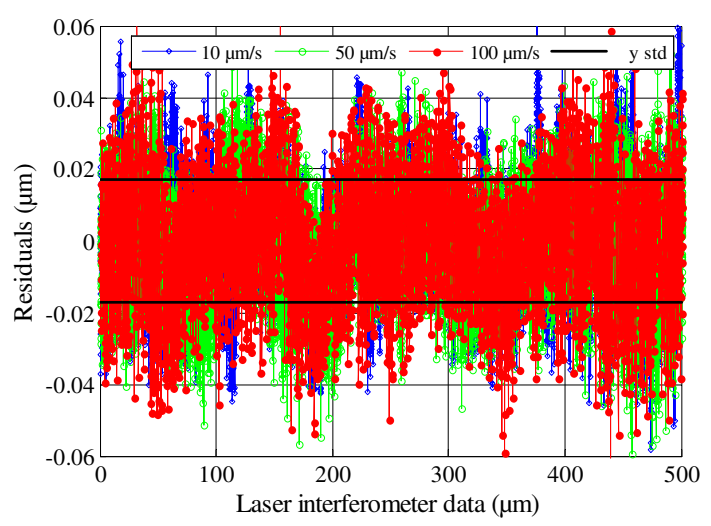

Fig. 2. (a) In-situ calibration of the optical confocal chromatic probe of $350 \mu \mathrm{m}$. Evolution of the linear residuals versus the displacement measured by the $z$-differential laser interferometer (three values of speed: 10, 50 and $100 \mu \mathrm{m} / \mathrm{s}, Y$-std represents the standard deviation which is less than $2 \mathrm{~nm}$ ), (b) In-situ calibration of the inductive probe on the smaller range of $100 \mu \mathrm{m}$; evolution of the nonlinear residuals according to the displacement of the zerodur table measured by the $z$-differential laser interferometer, (c) In-situ calibration of the inductive probe on the medium range of $0-500 \mu \mathrm{m}$; evolution of the nonlinear residuals versus the displacement measured by the $z$-differential laser interferometer (three values of speed: 10,50 and $100 \mu \mathrm{m} / \mathrm{s}, y$-std represents the standard deviation which is less than $20 \mathrm{~nm}$ ).

between $\pm 18 \mathrm{~nm}$. The amplitude of these errors affects the capability of the machine, by increasing the total uncertainty. The effect of the speed is also investigated with 10 , 


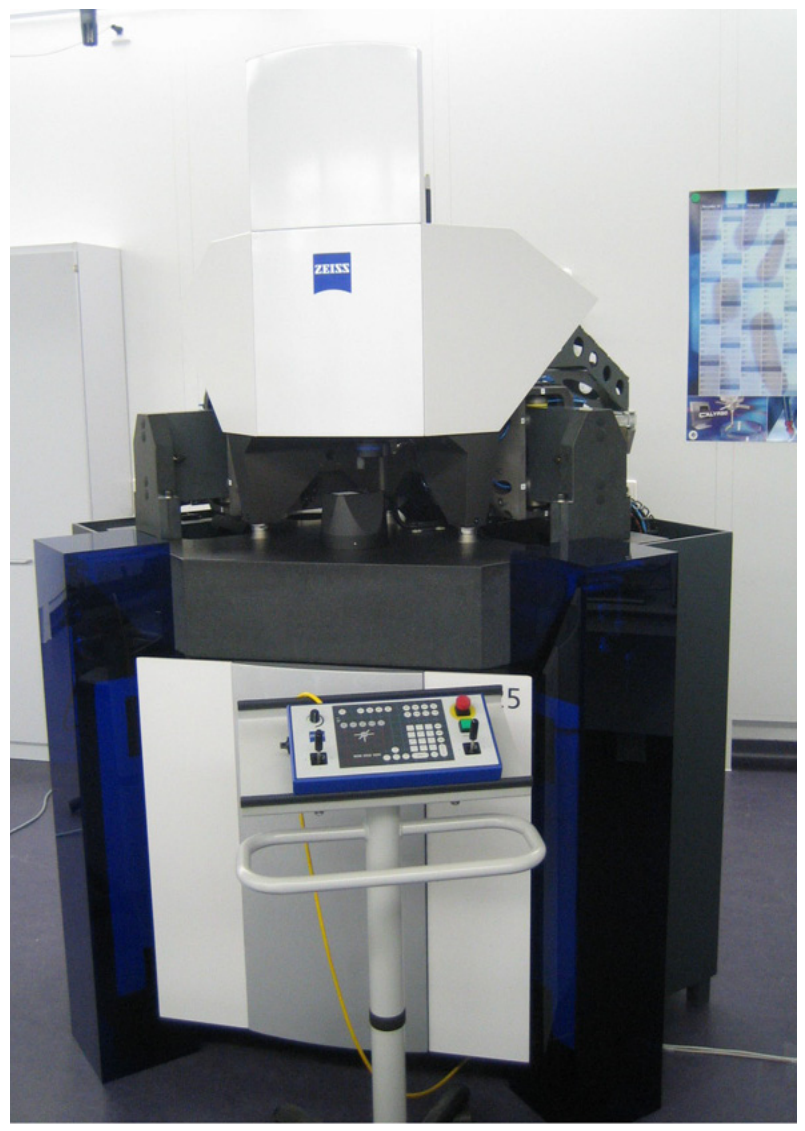

Fig. 3. Photograph of both VSL and SMD F25 apparatus.

50 and $100 \mu \mathrm{m} / \mathrm{s}$ and reveals that the measurement is not influenced by the speed.

\section{VSL and SMD apparatus, ZEISS F25}

The ZEISS F25 microCMM ( $\mu$ CMM) development is the fruit of a cooperation between VSL, The Eindhoven University of Technology and ZEISS. The VSL and SMD Zeiss F25 $\mu \mathrm{CMMs}$ are both built on a highly accurate, air-bearing kinematics structure with linear drives and an active air damping base [11]. Ultra-precise glass-ceramic scales are used as the length measuring systems for all three axes with a resolution of $7.8 \mathrm{~nm}$ for the VSL machine and $1 \mathrm{~nm}$ for the SMD machine which is a later F25 generation. The measuring range is $100 \times 100 \times 100 \mathrm{~mm}^{3}$ for VSL machine and $130 \times 130 \times 100 \mathrm{~mm}^{3}$ for the SMD machine (Fig. 3).

The design applies largely the Abbe principle to reach higher accuracy. This is realized by using additional intermediate bodies in $x$ - and $y$-direction as compared to conventional CMM designs. Each intermediate body caries a crossing beam mounted perpendicular to the direction of movement of the intermediate body. The intermediate bodies are supported on orthogonal beams ( $x$ and $y$ ), connected to the probe by a moving platform. The beams move through their respective intermediate bodies, each carrying a measuring head for the line scales. These bodies can move along their respective guiding beams which are mounted on a base. From this base a granite table for the products to be measured is suspended. In the mid-plane of the measurement volume the line scales are pointing directly to the probe meaning the first order Abbe error is 0. A small Abbe error exists when moving in $z$-direction out of the mid-plane of measurement.

The error model of the F25 is described in more detail in the thesis of Vermeulen [11]. To improve the uncertainty of the F $25 \mu \mathrm{CMM}$ the error sources need to be quantified and corrected. Without corrections the volumetric uncertainty of the F25 is estimated to be about $1.63 \mu \mathrm{m}$. The uncertainty after correction depends on the capability to quantify these error sources, and how stable these error sources appear to be over time. To reduce the geometrical errors calibration methods were developed [12] in cooperation between VSL and ZEISS. A CAA (Computer Added Accuracy) model of the F25 was implemented in the controller of the $\mu \mathrm{CMM}$. The CAA model includes the linearity, rotational and squareness errors, but the straightness deviations are not corrected for. The residual errors have been determined with respect to the CAA corrections and include a drift of the machine over a 3.5 year period by comparing the calibration data of 2012 and 2008. The results were quite similar to the drift over $\sim 1$ year determined in 2007.

When applying the corrections it can be seen that the volumetric uncertainty of the VSL-F25 is in worst case $\sim 400 \mathrm{~nm}$ over its full measuring range of $100 \times 100 \times$ $100 \mathrm{~mm}^{3}$, and $55 \mathrm{~nm}$ when measuring in a smaller measuring volume of $20 \times 20 \times 20 \mathrm{~mm}^{3}$. The main contributions to the uncertainty are the uncorrected straightness deviations.

In order to reduce the uncertainty caused by the geometrical errors further, a virtual CMM model of the VSLF25, used for the task-specific uncertainty calculation will include the residual deviations as a function of the machine coordinates and will be able to correct for them. This is especially relevant for the straightness deviations. A much lower uncertainty, especially for longer ranges will then be realized.

The F25 $\mu \mathrm{CMM}$ usually is equipped with an optical ViSCAN vision probe which is used as an optical probe for 2D measurements [13], and a tactile microprobe.

The uncertainty due to thermal drift strongly depends on the imperfection of the thermal loop [12], which has been designed to be negligible by using materials with different expansion coefficients. However there is also an uncertainty on the thermal expansion coefficients. The thermal errors are minimised by:

- the machine is shielded from the operator's thermal radiation and driven by remote motion control;

- heat production in the drives is small due to a high motor constant and a small electrical resistance;

- room temperature fluctuations are limited to the laboratory conditions: $\pm 0.1 \mathrm{~K}$.

Based on the geometry of the thermal loop and an estimated $5 \%$ deviation in the thermal expansion coefficients the uncertainty has been determined as a function of the 


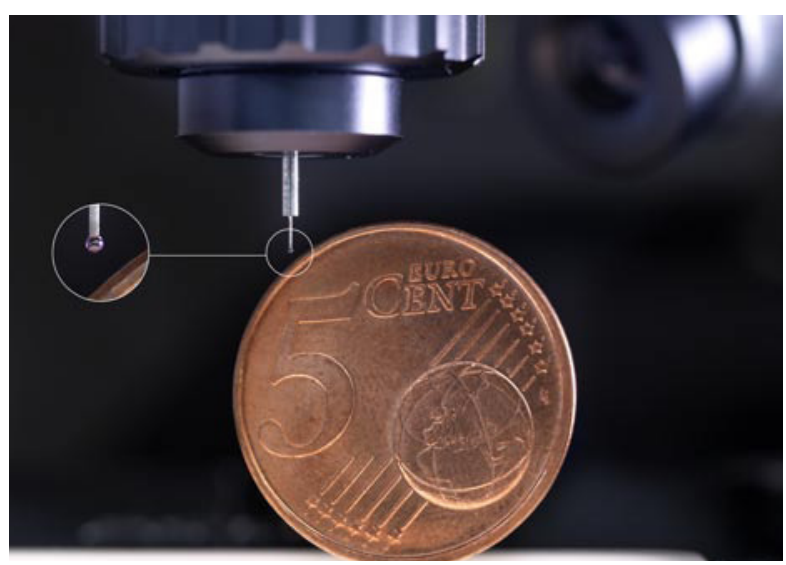

Fig. 4. Tactile probe of the F25 (IMT).

temperature variation during the measurement. According to the laboratory conditions, it is possible to get this level down to $10 \mathrm{~nm}$.

Usually the measurements time is short enough that the contribution due to thermal effects becomes negligible. For longer measurement times special care has to be taken to obtain the best thermal stability of the F25. The evaluation of the thermal drift for longer measurement times is experimentally done by using an invar ball plate and reveals that the thermal drift is less than $1 \mathrm{~nm} / \mathrm{min}$, $0.8 \mathrm{~nm} / \mathrm{min}$ and $1.9 \mathrm{~nm} / \mathrm{min}$, respectively for the $x, y$ and axes.

\section{VSL and SMD F25-IMT probe}

The IMT tactile probe of the F25 consists of a very thin shaft, carrying the ruby contact ball and allows probing in 3D. Ball diameters go down to $120 \mu \mathrm{m}$. Shaft diameters go down to $100 \mu \mathrm{m}$. With a free shaft length up to several millimetres, it is possible to measure inside small deep-lying structures. The stylus permits measurements in both single point and in scanning mode. The shaft is fixed in the centre of a $6.5 \times 6.5 \mathrm{~mm}^{2}$ silicon membrane with integrated piezo-resistive elements. The measuring forces are in the range of $0.5 \mathrm{mN}$. The exact probe ball diameter is determined by a measurement on a calibrated reference sphere. The $x, y$ and $z$ deflection signals of the probe are combined with the signals from the $x, y$ and $z$ scales to generate measurement results (Fig. 4).

\subsection{Uncertainty contribution due to the probe}

In addition to machine geometrical errors, the probe gives an important contribution to the overall uncertainty of the F25 machine. To ensure the measurement of a dimension (length), many configurations should be distinguished.

For the case of a planar-like structures/high aspect ratio structures without real slopes, only sharp edges (examples are holes, rings and plugs) two cases can be distinguished: uni-directional and bi-directional measurements. For uni-directional measurements, the two points which
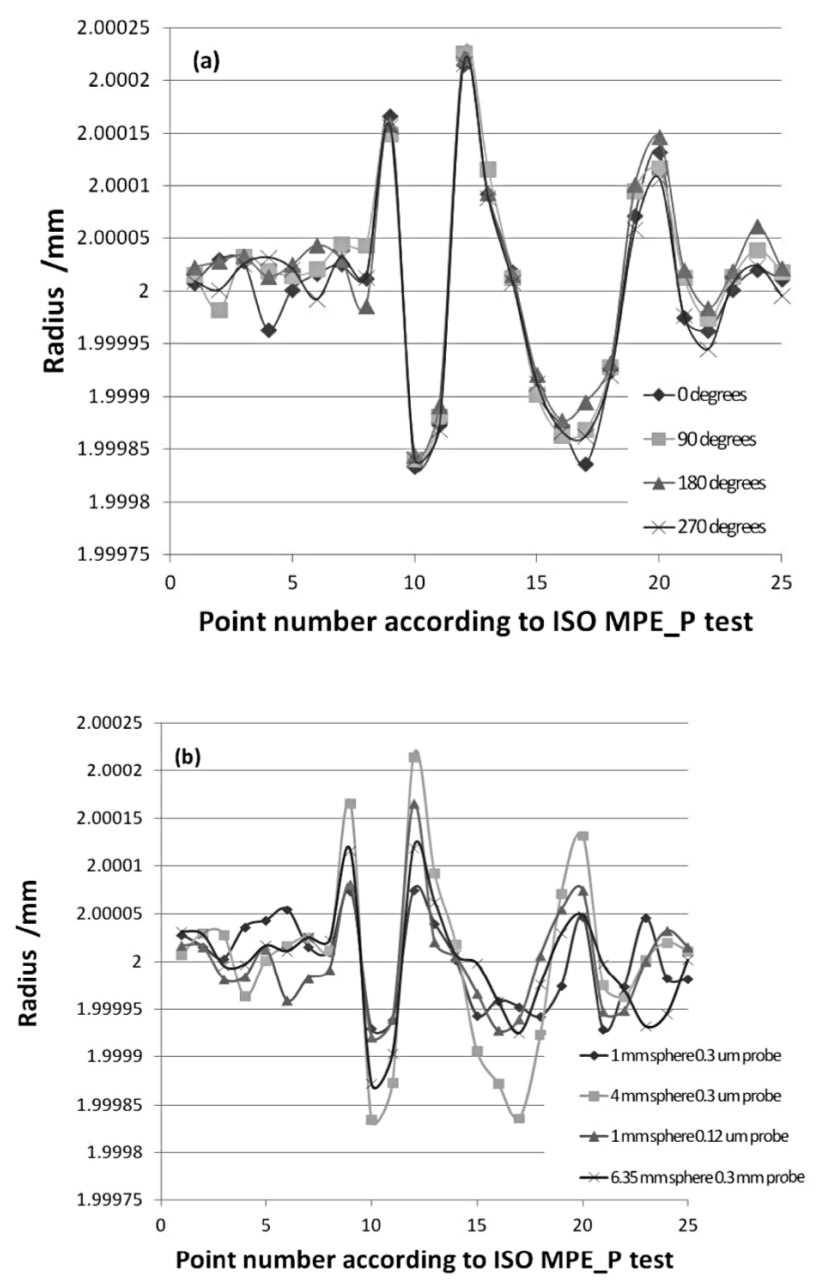

Fig. 5. Points measured on sphere according to ISO-10360.

determine the length are probed from the same side. Therefore the geometry of the probe ball does not play a role, since it gives a systematic deviation which is identical for both points and therefore cancels out when the length is determined. The repeatability of the probe determines the uncertainty and was experimentally estimated to $9 \mathrm{~nm}$.

For bi-directional measurements, the dimension (length) is determined by probing from two different sides, and both the diameter and form error of the micro-probe sphere needs to be known, since a correction is necessary. The uncertainty $(1 \sigma)$ relative to the diameter calibration is of $15 \mathrm{~nm}$, and the $1 \mathrm{~s}$ uncertainty contribution due to the roundness is estimated to $7 \mathrm{~nm}$. The estimated uncertainty for both cases are summarised in the Table 1.

For the case of structures with slopes (example of balls and lenses), the stiffness of the IMT probe is different for $x, y$ as compared to $z$-axis. When probing on a slope therefore the stiffness depends on the probing vector, and causes an anisotropy in the probing [14,15]. The anisotropy of the micro-probe leads to an additional uncertainty which depends heavily on the actual slopes of the structure and which is estimated to be $250 \mathrm{~nm}$ worst case, based on MPEP (Maximum Permissible Error of 
Table 1. Uncertainty due to probing.

\begin{tabular}{ccc}
\hline & \multicolumn{2}{c}{ Uncertainty } \\
\hline Repeatiblity $(1 \sigma)$ & 0.009 & \\
Reference sphere & 0.015 & \\
calibration $(k=1)$ & 0.007 & \\
Roundness deviation & Bi-directional & Uni-directional \\
& 0.019 & 0.009
\end{tabular}

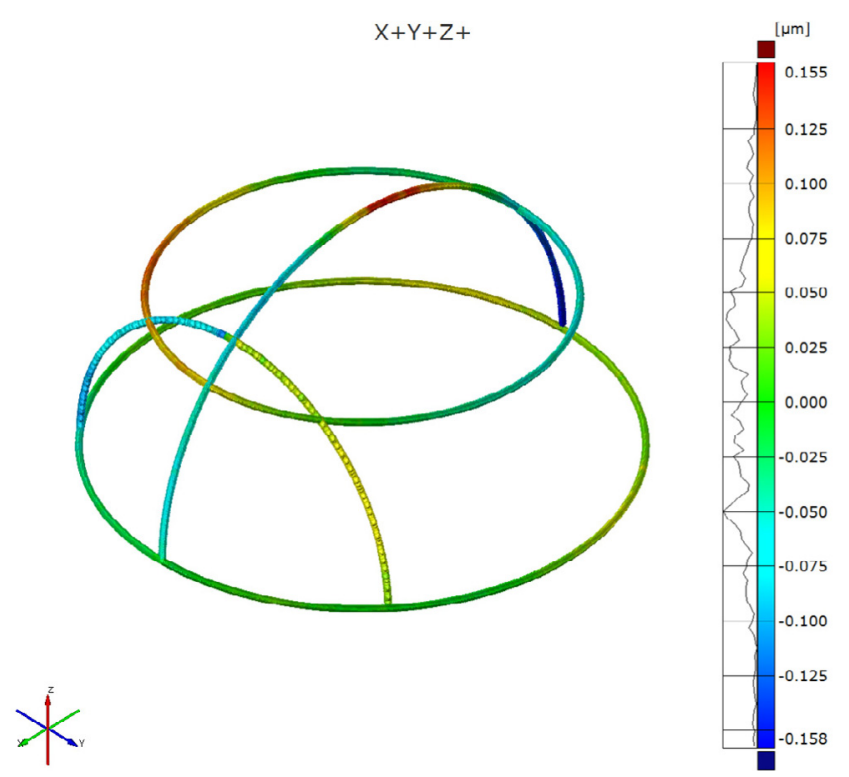

Fig. 6. Scanning behavior of the F25 according to the ISO10360-4 acceptance test.

Probing) test according to ISO ISO-10360. Correction for the anisotropy can be done by measuring at different orientations or off-line corrections, reducing the uncertainty contribution from 250 to $50 \mathrm{~nm}$.

\subsection{Effects when scanning}

Measuring an object can be done by static probing, were every point is individually probed, or in scanning mode, were the probe remains in contact with the surface. Scanning measurements have the advantage of obtaing more datapoints in the same amount of time. This reduces the effect of local variation, such as roughness, and improved statistics. However, when scanning a surface additional effects may appear which can contribute to the uncertainty of the probe. For this reason, a scanning test on a sphere was performed based on ISO-10360, Figure 6, as can been seen a hysteresis like effect occurs when scanning over the top of a sphere or lens. Knowing these effects makes it possible to choose measurement strategies which reduces these effects to levels were they can be neglected.

\subsection{Uncertainty evaluation for the measuring of the diameter of rings and plugs}

Since the ring and plug present a planar-like structure the probing anisotropy can be neglected. Furthermore it is an
Table 2. Evaluation of the uncertainty.

\begin{tabular}{ccc}
\hline $\begin{array}{c}\text { Simplistic uncertainty budget } \\
\text { calibration of a ring and plug }\end{array}$ & $\begin{array}{c}3 \mathrm{D} \\
(\mu \mathrm{m})\end{array}$ & $\begin{array}{c}2 \mathrm{D} \\
(\mu \mathrm{m})\end{array}$ \\
\hline Geometrical volumetric uncertainty $(k=1)$ & 0.027 & 0.016 \\
Probe error bi-directional $(k=1)$ & 0.019 & 0.019 \\
Finite stiffness $(1 s)$ & 0.005 & 0.005 \\
Thermal errors $(1 s)$ & 0.002 & 0.002 \\
Total uncertainty of a length $(1 s)$ & 0.034 & 0.025 \\
Total expanded uncertainty $(k=2)$ & 0.067 & 0.051 \\
\hline
\end{tabular}

almost 2D measurement and not a 3D. Obviously the ring and plug need to be positioned in its optimal measurement position, so in the Abbe plane of the F25. The uncertainty for the VSL-F25 is given in Table 2. The uncertainty for the roundness measurement is quite similar.

\subsection{Uncertainty evaluation for the measuring the diameter of a sphere and lens}

A sphere and lens present not a planar-like structure and the anisotropy of the probe has to be considered. When a measurement strategy is chosen with evenly distributed points on the sphere the effect of the anisotropy of the probe cancels out for the diameter measurement due to the symmetry of the anisotropy. The $67 \mathrm{~nm}$ is a bit higher than the uncertainty of $50 \mathrm{~nm}$ which was the goal and can be improved by considering additional corrections, especially for the straightness errors as can be seen in Table 2 .

The uncertainty of the sphericity is dominated by the anisotropy. So a rough worst case estimate is an expanded uncertainty of $250 \mathrm{~nm}$ for sphericity when no corrections are applied. For a lens, which can be considered as a segment of a sphere, the actual uncertainty due to the anisotropy depends on the slope of the lens where the measurements are taken.

\section{SMD confocal probe on ZEISS F25}

Even though the tactile probe of the Zeiss F25 is more accurate than the vision probe, it is one of the dominant contributors to the overall measurement uncertainty. This is the main reason why a tilt-robust optical probe is being developed for the F25. Apart from improving the overall measurement uncertainty of the F25, the addition of an optical distance probe enables measurement of soft or very delicate surfaces.

The optical probe uses the differential confocal method to measure the distance to the surface under test, in combination with an aperture monitoring system to compensate for tilt dependent errors. Basically, this is the same principle as used for the optical probe in NANOMEFOS, with the exception that the probe for the F25 will not include a guidance and interferometer system to extend the measurement range of the probe, since here the F25's $Z$-axis drive can be used to bring the probe into range. Furthermore, the probe's properties are optimized for the 
F25, the optical system and mechanics are redesigned to match the F25's performance and mechanics, and the optical design incorporates improvements to enable for better compensation of tilt dependent error.

The most important property that makes this probe suitable for use with the F25 is that it delivers tiltrobust measurements with nanometre level uncertainty. The performance of traditional optical distance probes with nanometre resolution, including chromatic confocal and differential confocal probes as well as those based on interferometric principles, is degraded by tilt dependent errors of at least many hundreds of nanometres and often micrometers. These errors in optical distance probes can be caused by three different contributions, namely due to:

- vignetting of returning light;

- inherent tilt sensitivity of the measurement principle;

- aberrations in the system, most notably the objective lens.

Hence, without proper measures, an optical distance probe cannot deliver the measurement uncertainty required to match the performance of the F25. To enable tiltrobust measurements with nanometre-level uncertainty, the sources of the tilt dependent errors are minimized, and the errors that nonetheless remain are subsequently corrected for.

The three measures taken to minimize tilt dependent error are:

- under-filling the objective lens so that even at maximum tilt vignetting is negligible;

- optimizing the $Z$-axis drive parameters of the differential confocal system to minimize its inherent tilt sensitivity;

- minimizing the aberrations in the optical system, including a custom designed high NA objective lens.

The remaining tilt dependent error is compensated by sampling $10 \%$ of the returning laser beam and measuring through which part of the aperture the light returns with a PSD (Position Sensitive Detector) and using the measured aperture position to correct the distance measurement using a lookup table.

The linearization and correction of the signals have to be performed in real-time by a signal processing unit of which the hardware and firmware is designed for this probe. To allow scanning measurements at high scan speeds, data-age is kept below $50 \mu$ s at least. The signal processing unit has to be placed outside of the F25's volume, so that dissipation in the electronics will not affect measurement conditions. Only the primary amplifier stages and a minimum of passive electrical components are located at the probe underneath the F25 $Z$-axis drive, to minimize power dissipation to a few $\mathrm{mW}$.

The objective of the probe is removable and the probe is placed such that both the original tactile and vision system of the F25 can still be used. The approximate layout and volume envelope can be seen in Figure 7.

The probing laser beam is focused down to a spot diameter of about a micrometer. The $Z$-axis capturing range will be a few $10 \mu \mathrm{m}$ to allow for the overshoot of the F25
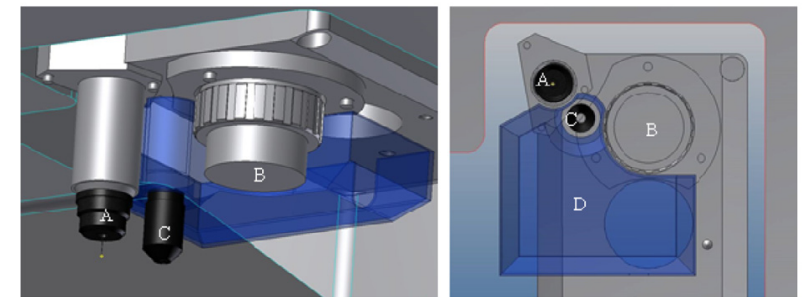

Fig. 7. Approximate volume envelope (D) for the probe from operator point of view (left) and from the bottom (right), the tactile probe (A), F25 vision system (B) and optical probe's objective (C) are indicated.

Table 3. Overview of requirements for the optical probe.

\begin{tabular}{cc}
\hline Probe property & Requirement \\
\hline Measurement range & $\sim 4 \mu \mathrm{m}$ \\
Capture range & $\sim 60 \mu \mathrm{m}$ \\
Measurement uncertainty & $<40 \mathrm{~nm}$ \\
at acceptance angle $(2 \sigma)$ & $<1 \mathrm{~nm}$ \\
Resolution & $5^{\circ}$ to $10^{\circ}$ \\
Acceptance angle & $>20 \mathrm{kHz}$ \\
Sample rate & $\sim 1 \mu \mathrm{m}$ \\
Spot size & $\sim 1.5 \mathrm{~mm}$ \\
Probe stand-off & $<50 \mathrm{~nm} / \mathrm{K}$ \\
Thermal sensitivity &
\end{tabular}

after the surface under test comes into focus, while the $Z$-axis measuring range will be about $4 \mu \mathrm{m}$, the resolution is better than $1 \mathrm{~nm}$, the maximum angle of the measured surface with respect to the plane perpendicular to the measuring axis of the probe $5^{\circ}$ to $10^{\circ}$ and the $k=2$ uncertainty below $40 \mathrm{~nm}$ at the maximum surface slope angle.

The main advantage of this probe is the contactless measuring mode, combined with sub-nanometre resolution and a very low measurement uncertainty. This is combined with a very good lateral $(X-Y)$ resolution. This new probe can allow making measurements with uncertainties significantly below the uncertainties of measurements obtained with the original tactile probe and this on hard surfaces, as with the tactile probe, but also on soft surfaces and highly delicate surfaces. An overview of the properties of the probe is given in Table 3.

\section{METAS $\mu$ CMM}

The METAS $\mu$ CMM (Fig. 8a) has been especially designed to measure micro-parts with an ultra-high accuracy [16]. It is composed of a $3 \mathrm{D}$ tactile probe head and a motion stage. The ultra-precision CMM motion stage has vacuum preloaded air bearings driven by Lorenz actuators and its motion is controlled by an interferometric position measurement. The special slide configuration with two wedges makes the stage very compact and stiff. A pneumatic weight compensation system minimises the heat generation for lifting up the heavy stage in the $z$-direction. Therefore, the electric power consumption of the actuator 


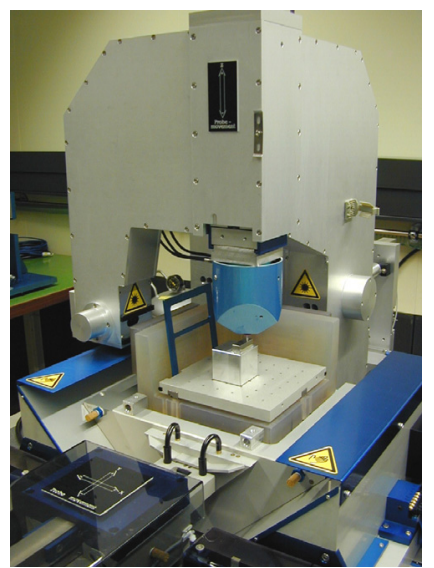

(a)

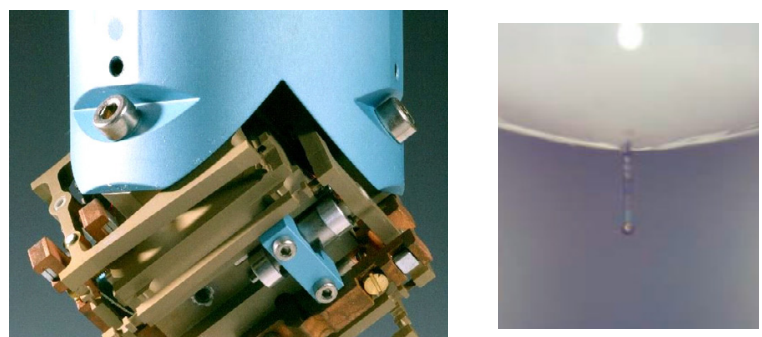

(a)

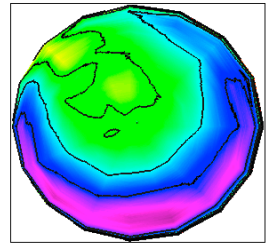

(b)

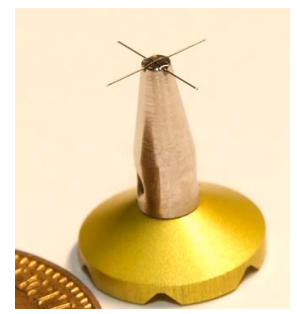

(c)

Fig. 9. METAS microprobe. (a) Photograph and form error of a tungsten probe. (b) Diameter: $67.9 \mu \mathrm{m}$. Sphericity: $3.7 \mu \mathrm{m}$. (c) Photograph of a quad probe: $4 \times 0.125 \mathrm{~mm}$ sapphire spheres glued on tungsten shafts.

(b)

Fig. 8. METAS CMM apparatus and tactile microprobe. (a) Photograph of the METAS ultra-high precise CMM. (b) Photograph of the prototype 3D touch probe head.

system for all axes remains below $0.5 \mathrm{~W}$. The working volume of the stage is $90 \times 90 \times 38 \mathrm{~mm}^{3}$.

During measurements the probe head is standing still while the stage moves the workpiece in all directions around the probe. The workpiece sits in a Zerodur cube corner with three perpendicular flat mirrors on the outside which forms the reference coordinate system. The displacement of all axes is measured with no Abbe offset since all three interferometer beams point to the centre of the probing sphere.

The probe head is linked to the interferometers through the metrology frame. As the frame has to be rather large to go around the stage and to hold the $z$-axis interferometer from below, the frame is very sensitive to temperature variations which will cause drift. To reduce temperature gradients the frame is made out of aluminium, which makes the thermal behaviour quite predictable. Additionally, the frame is thermally shielded by a thick aluminium housing. In future various contributions to drift will additionally be compensated applying numerical models. Finally, thanks to a special cabin around the CMM and its location in a stable temperature controlled clean room laboratory in the basement of METAS, the temperature changes of the frame remain below $1 \mathrm{mK}$ during a measurement.

Two video microscopes and a logarithmic joystick complete the user-friendly interface, making the positioning of the probe on the small sample features as easy as for conventional CMMs.

\section{METAS 3D probe head}

The 3D touch probe head has a particular parallel kinematic structure that exhibits very week and isotropic probing forces, and minimizes the moving mass within the probing system (Fig. 8b). Based on three parallelograms with flexure hinges, this structure leaves the probing sphere exactly three degrees of freedom. While all rotational movements are blocked, the translational motion is separated into its $x y z$-components, which are measured by three inductive probes. Due to the special orientation of the probe head coordinate system all axes are identical with respect to gravity and have equal probing forces in all directions. The main part of the structure is manufactured out of a single piece of aluminium using electrodischarge machining. The flexure hinges have a thickness of only $60 \mu \mathrm{m}$ resulting in a stiffness of $20 \mathrm{mN} / \mathrm{mm}$. Due to this very low stiffness, the deformation caused by gravity needs to be compensated. For this purpose an adjustable system based on permanent magnets, that introduces a gravitation compensation almost independent to the probe deflection, was built into each axis of the probe head.

The measurement range is $\pm 0.2 \mathrm{~mm}$ while the mechanical limits allow a tip deflection of $\pm 0.5 \mathrm{~mm}$ in all directions. The effective moving mass is only $7 \mathrm{~g}$. A small moving mass is important to keep the dynamic contact forces low while maintaining reasonable approaching speeds.

The magnetic holding of the probing element allows an easy tip replacement and cleaning. Additionally, it acts also as mechanical fuse in case of a collision. This highly sensitive device is therefore quite robust and its handling remains easy. Many different probes with sphere diameters from $0.06 \mathrm{~mm}$ to $1 \mathrm{~mm}$ and with different geometries can be used to target almost any measurement task (Fig. 9) [17].

All machine corrections such as for the laser wavelength, the reference mirror flatness, the angles between the reference mirrors, and even the probing sphere shape deviation for which a particular in situ procedure was developed [18], were fully characterized and implemented in the machine controller software developed at METAS. This low level software does not only control the probing procedure for single-point measurements but also allows scanning data acquisition without compromising the measurement accuracy [19]. The high level software QUINDOS (Messtechnik Wetzlar GmbH) is used to provide easy programming of measurement sequences and complex data evaluations.

In order to prove the $\mu \mathrm{CMM}$ accuracy, several acceptance tests, analogue to those for conventional CMMs, were performed. Referring to the standard ISO 10360-2, seven Zerodur ball bars were measured parallel to the axis 


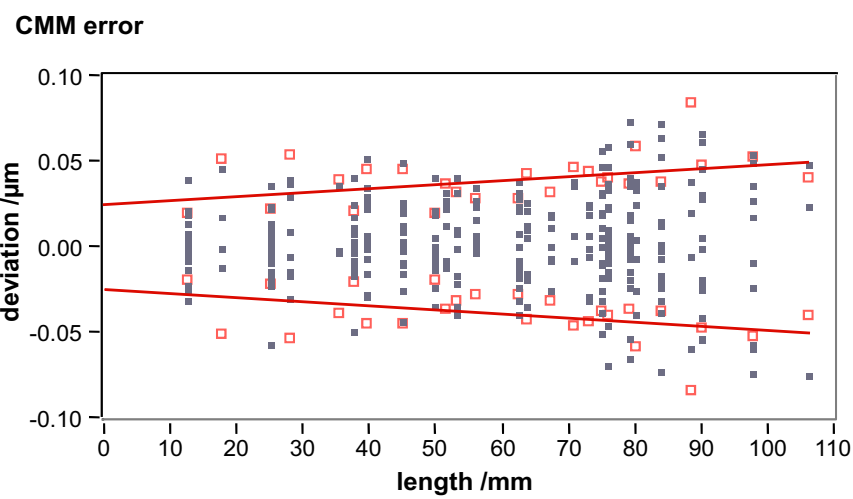

Fig. 10. The combined uncertainty estimation $(k=2)$ for the METAS $\mu$ CMM estimated from a ball plate measurement.

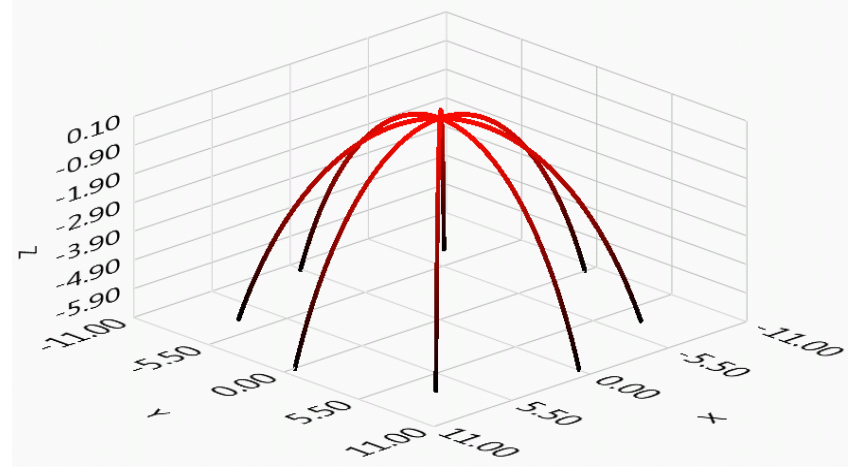

Fig. 11. 4 profiles scanned on the aspheric lens.

and in diagonal directions within the $\mu \mathrm{CMM}$ measuring volume. Similarly, an invar ball plate with 25 spheres was measured following the DKD 2617 guidelines. The combined uncertainty estimation $(k=2)$ achieved during both of these tests was $0.027 \mu \mathrm{m}+\mathrm{L} / 4000 \mu \mathrm{m} / \mathrm{mm}$ (Fig. 10).

The unique features and the precision of this machine render it very attractive for measuring among others optical components, especially those with steep slopes where traditional optical measurement techniques show their limits.

To illustrate the machine's possibilities, an aspheric lens $\varnothing=23 \mathrm{~mm}$ with slopes up to $60^{\circ}$ was scanned along 4 profiles using the METAS $\mu$ CMM fitted with a probe $\varnothing=0.3 \mathrm{~mm}$ (Fig. 11). The aspheric form with fixed aspheric parameters was then fitted to all the data points. The residual form error exhibits deviations of only $\pm 50 \mathrm{~nm}$. The main contribution (low frequency) is attributed to the residual form error of the lens itself, and for a small contribution (estimated to be less than $\pm 15 \mathrm{~nm}$ ) to the residual calibration error of the probe shape. The high frequency noise of about $\pm 8 \mathrm{~nm}$ on each profile is attributed to the surface roughness of the probe and the lens, and to the electronic noise.

\section{TNO measuring apparatus}

The NANOMEFOS machine [20,21] (Fig. 12) was specifically designed for non-contact measurement of aspherical

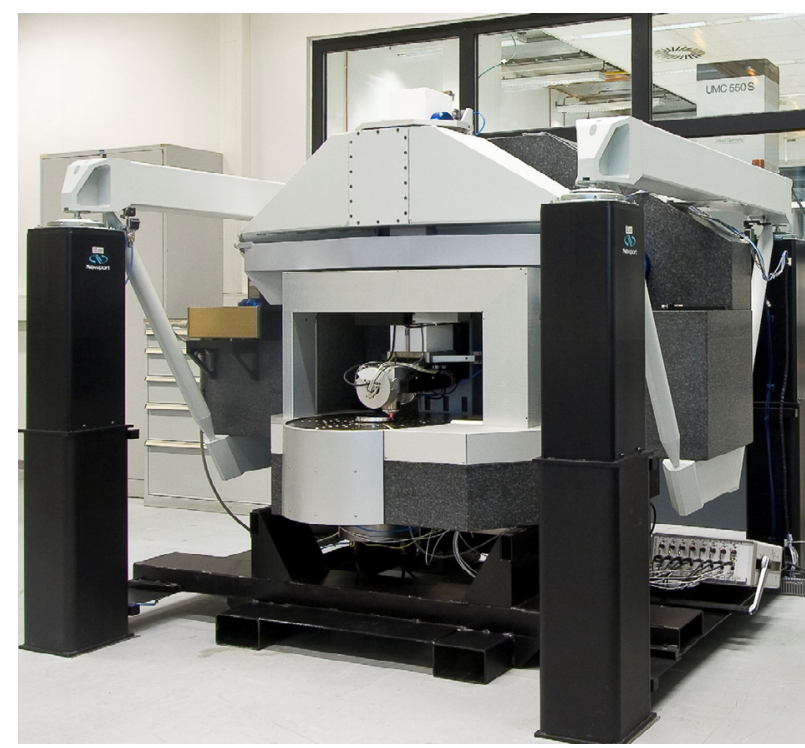

Fig. 12. NANOMEFOS non-contact measurement machine.

and freeform optics. As these surfaces are always (moreor-less) rotationally symmetric, a cylindrical setup allows for less moving axes and higher measurement speed, compared to orthogonal setups. To allow for measuring slopes from $-45^{\circ}$ concave to $+45^{\circ}$ convex, the optical probe can be tilted such that it is aligned perpendicular to the bestfit asphere of the surface. The machine has a measurement volume of $\varnothing 500 \times 100 \mathrm{~mm}$, and an uncertainty in the order of $10 \mathrm{~nm}$. For the largest surfaces, obtaining 4 million points (0.2 mm point density) takes about $15 \mathrm{~min}$.

The NANOMEFOS machine presents a cylindrical concept and resembles a giant CD-player (Fig. 12). The product is mounted on an air-bearing spindle, which is rotating continuously at for instance $1 \mathrm{rev} . / \mathrm{s}$. A custom non-contact optical distance probe is mounted on a rotation axis $(\Psi$-axis $)$ which positions it perpendicular to the rotationally symmetric best-fit of the product. The probe can be moved in radial and vertical direction by the $R$ and $Z$-stage, respectively. This way, the surface height profiles are measured, either by (multiple) circular tracks or by a continuous spiral track.

The machine is equiped by an optical probes with a focal depth of a few micrometres when nanometre resolution is required. Keeping the probe in focus by actuating the $R$ and $Z$-stage requires large accelerations of these heavy stages, resulting in undesirable dynamics. To avoid this, a probe with $5 \mathrm{~mm}$ range was developed [22]. This way the stages can be kept stationary, even when measuring a freeform track. This reduces the dynamically moving mass from a few hundred $\mathrm{kg}$ for the stages to only about $50 \mathrm{~g}$ of the probe objective lens. This improves system dynamics and allows for maintained high scanning speed for freeform surfaces.

The measurement uncertainty is mainly determined by the metrology loop between the probe and the product. By applying a separate metrology system relative to which the probe and product position are measured, the metrology loop becomes much shorter than the structural loop. 


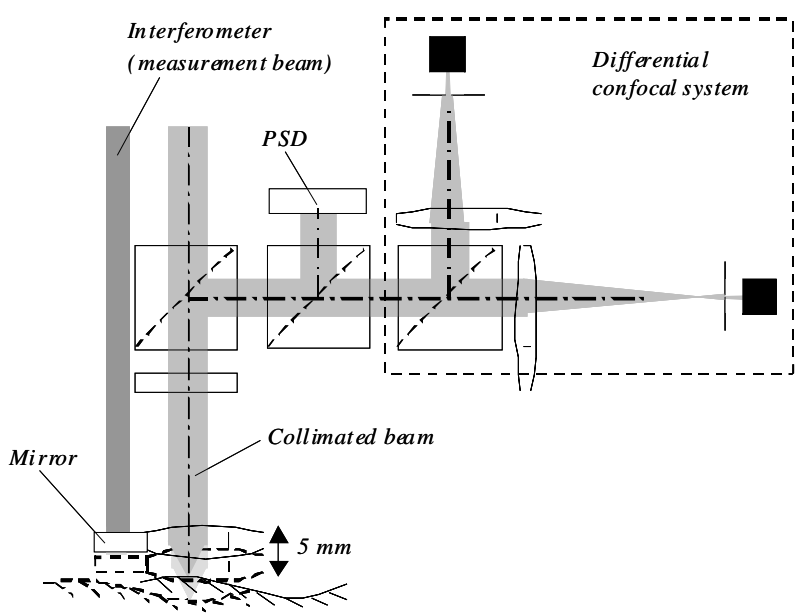

Fig. 13. Schematic of dual stage probe (differential confocal with interferometer) and aperture position monitor using PSD for tilt dependency correction.

Hereby most of the stage errors are eliminated from the metrology loop.

With the probe more-or-less perpendicular to the surface, the error sensitivity is different compared to orthogonal machine setups. As the surfaces are smoothly curved, the tangential error sensitivity is reduces, which reduces the metrology problem to a 2D-problem, since all sensitive directions are in the plane of motion of the probe. Hence, a metrology system consisting of interferometers, capacitive probes and a Silicon Carbide metrology frame provide a short and stable loop in the measurement plane. A high-stability parallel air-bearing stage setup with split position and preload frames provides sub-micrometer and micro-radian $(\mu \mathrm{rad})$ repeatability in the other directions. When measuring a freeform surface, only the probe focusing mechanism is moving dynamically while measuring a circular track. The static and dynamic displacements of probe and product that occur during this measurement are recorded by the metrology system and can be compensated for in the (off-line) data-processing.

\section{TNO optical probe and evaluation}

\subsection{Description of the TNO optical probe}

The TNO optical probe measures the distance between the $\Psi$-axis rotor and the surface under test, with an uncertainty of $10 \mathrm{~nm}$ when the surface is perpendicular to the probe and $35 \mathrm{~nm}$ when the surface is at $5^{\circ}$. A dual stage design is applied, which has $5 \mathrm{~mm}$ range, nanometre resolution and $5^{\circ}$ uni-directional acceptance angle. The design consists of a compact integration of the differential confocal method with an interferometer (Fig. 13). The focusing objective is positioned by a flexure guidance with a voice coil actuator. A motion controller finds the surface and keeps the objective focused onto it with some tens of nanometres servo error.

A Position Sensitive Detector (PSD) in the loop monitors the position in the aperture of the beam reflected
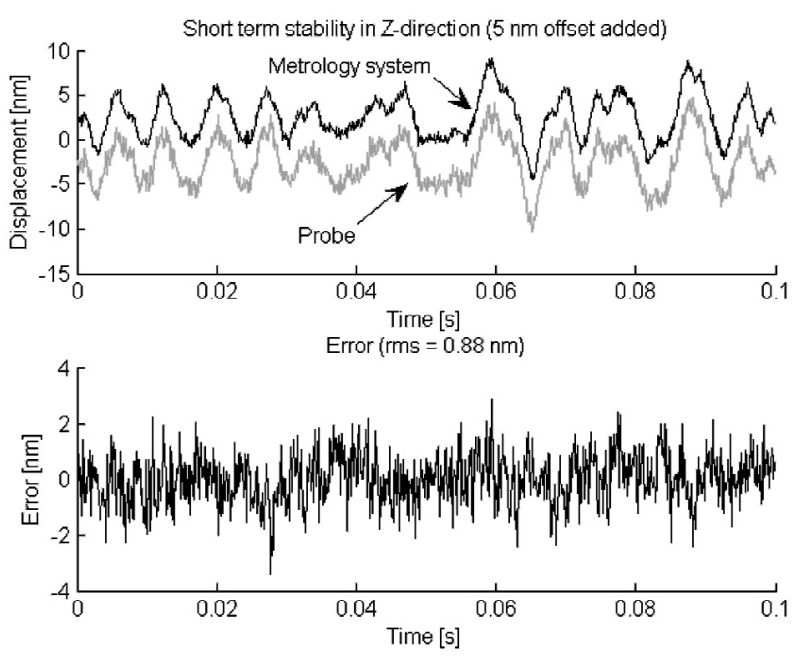

Fig. 14. Noise levl of the metrology loop (including the noncontact probe), showing the cancellation of most of the position errors by the separate metrology system.

by the surface, to enable compensation of the inclination dependent error that is inherent to the use of an optical probe [22].

The differential confocal method has been modelled, tested and optimized. The range of the system realized is $4 \mu \mathrm{m}$ and the noise level is $0.17 \mathrm{~nm}$. The repeatability over the full range is $4.1 \mathrm{~nm}$. Preliminary measurement results show a tilt dependency of about $550 \mathrm{~nm}$ at $5^{\circ}$, which is compensated to nanometre level uncertainty.

\subsection{Evaluation of the NANOMEFOS machine}

Assuming that the product position on the table is stable, the sum of the metrology loop and the optical probe should be constant. The displacement is measured by both the metrology system and the probe over a period of $0.1 \mathrm{~s}$. An offset of $5 \mathrm{~nm}$ has been added only for clarity. With all stages locked, including the spindle, a peak-to-valley motion in $z$-direction of $15 \mathrm{~nm}$ can be seen. The difference between the displacement measured by the metrology system and the probe is of $0.88 \mathrm{~nm}$ (Fig. 14).

For the evaluation of the traceability and uncertainty, tests done so far are the measurement of a $\varnothing 350 \mathrm{~mm}$ flat, which was calibrated to be $10 \mathrm{~nm}$ RMS, and measured to be $9 \mathrm{~nm}$ RMS by NANOMEFOS. A precision 1" sphere was measured to demonstrate the absolute radius of curvature accuracy, showing a repeatable difference of $125 \mathrm{~nm}$ (with the sphere calibrated to $0.1 \mu \mathrm{m}$ ). A $\varnothing 100 \mathrm{~mm}$ flat was measured interferometrically and on the NANOMEFOS with $2.5 \mathrm{~mm}$ tilt, representing an extreme freeform. With this tilt, the result shows $14 \mathrm{~nm}$ RMS (mostly astigmatism), where the flat itself is $5 \mathrm{~nm}$ RMS. A key feature here is the compensation of the inclination dependent errors as described in the next section. Based on the above results, the uncertainty is currently estimated to be better than $10 \mathrm{~nm}$ RMS for mild freeforms (up to $0.2 \mathrm{~mm}$ non-rotational symmetry). 


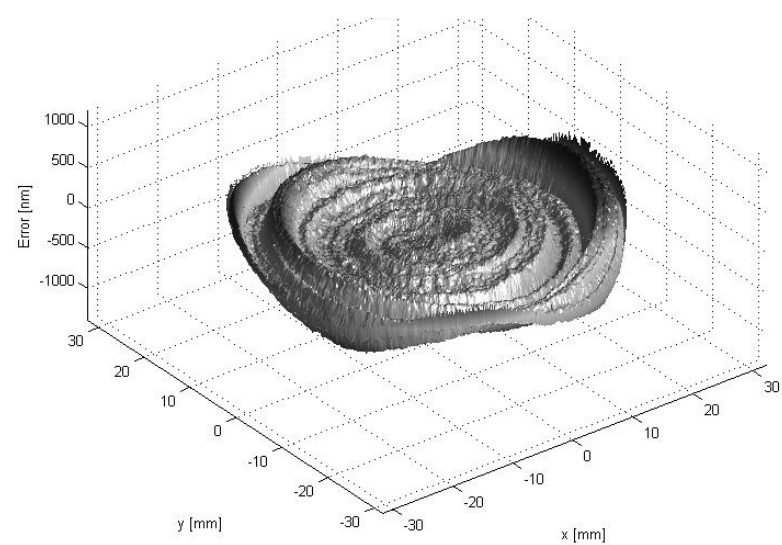

Fig. 15. Measured form error of an aspheric lens.

Table 4. Results of three consecutive measurements (RMS: Root Means Square, PV: Pick-to-Valley, $d R c$ : difference of the radius of curvature, the $d R c$ parameter illustrates the absolute radius measurement capability of the machine).

\begin{tabular}{lccc}
\hline & RMS $(\mathrm{nm})$ & PV $(\mathrm{nm})$ & dRc $(\mu \mathrm{m})$ \\
\hline Measurement 1 & 158 & 1077 & -5.79 \\
Measurement 2 & 158 & 1083 & -5.77 \\
Measurement 3 & 158 & 1096 & -5.74 \\
\hline
\end{tabular}

Figure 15 shows the typical measurement repeatability of a steep asphere of $58 \mathrm{~mm}$ diameter. This surface was measured with $0.2 \mathrm{~mm}$ point spacing, giving 300000 points, in about 9 min. Due the high point density, all mid-spatials resulting from the machining process clearly show. The results of three consecutive measurements are shown in Table 4. Here it can be seen that the RMS (Root Means Square) repeatability of the measured surface form error is below $1 \mathrm{~nm}$ RMS. The PV (PeaktoValley) varies slightly due to the steep edges. The fitted radius of curvature varies by approximately $50 \mathrm{~nm}$.

\section{IBSPE measuring apparatus}

The Isara 400 CMM features a measurement volume of $400 \times 400 \times 100 \mathrm{~mm}^{3}$. Three plane mirror laser interferometers are applied as measuring systems for the machine axes. The interferometers each measure against the sides of a mirror table, on which the work piece is mounted. These interferometers are mounted on a single body metrology frame, which also holds the probe system (Fig. 16). The laser beams are aligned to the probe tip and their mutual alignment does not change during movement of the axes, thus fulfilling the Abbe principle $[4,23]$ in $3 \mathrm{D}$ within the complete measuring volume. As a result, straightness errors and rotations of the three translation stages will have no first order influence on the measurement result. The influence of flatness and squareness errors of the three mirrors is eliminated by means of a series of on-machine calibration measurements. In the configuration of the translation axes, a machine concept was chosen in which the variable product mass does not

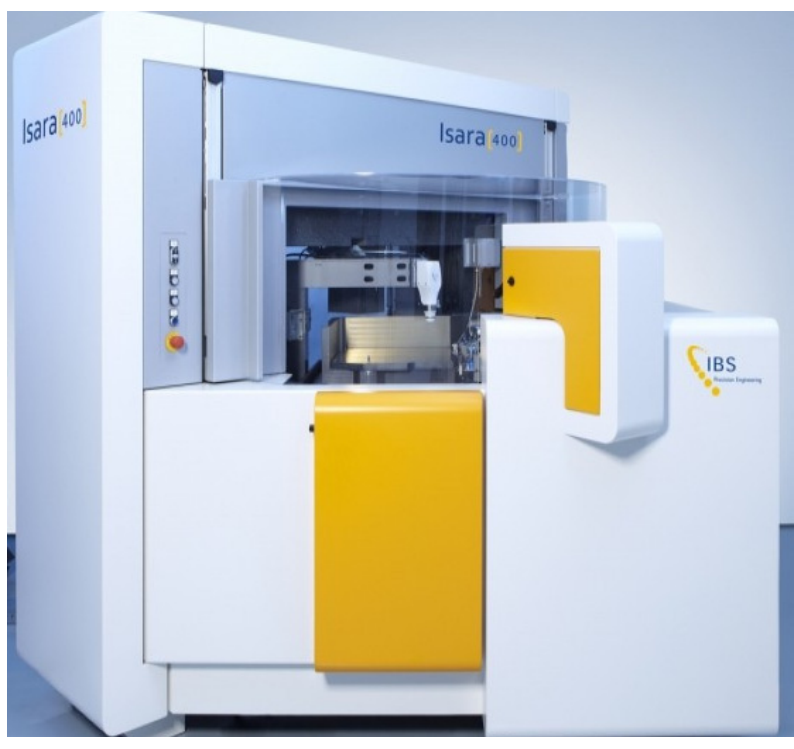

Fig. 16. Photograph of the complete ISARA 400 machine.

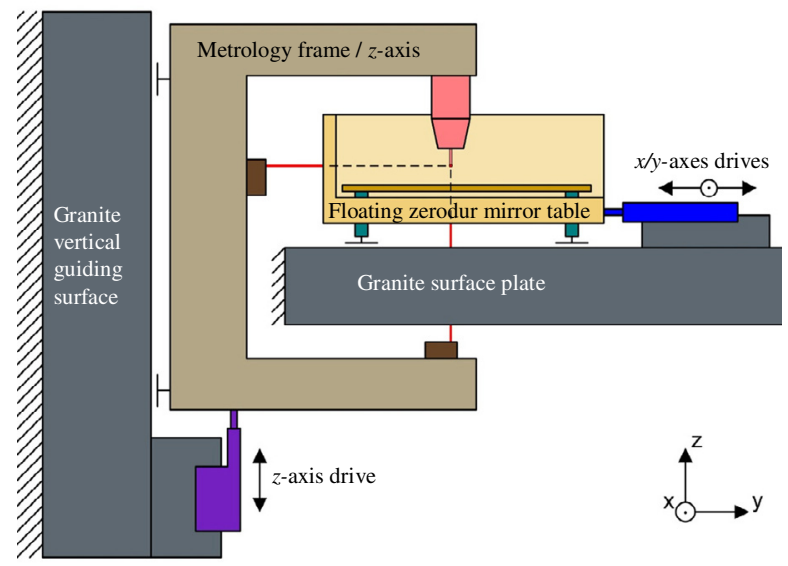

Fig. 17. 2D Design of the IBSPE Isara 400 CMM.

need to be moved vertically. The product is mounted on the mirror table, which moves only in $X$ - and $Y$-direction over a granite base plate, guided by air bearings in a "floating table" configuration. The complete metrology frame moves in $Z$-direction, with guiding provided by air bearings against a vertical granite surface (Figs. 16 and 17).

The function of the metrology frame, shown in Figure 17 , is to maintain the mutual position and alignment of the probe and the three laser interferometers with high stability. The metrology frame was designed as an assembly of hollow beams of silicon carbide $(\mathrm{SiC})$, resulting in a structure which is both stiff and lightweight, while also providing good thermal stability. The mirror table of the Isara 400 is a monolithic Zerodur part with three reflective sides. The mirror table is supported by three flat air bearings, whose preload is provided by the weight of the mirror table assembly with product. The work pieces are not placed directly onto the Zerodur, but onto a removable $\mathrm{SiC}$ product table, which serves as an interface between 

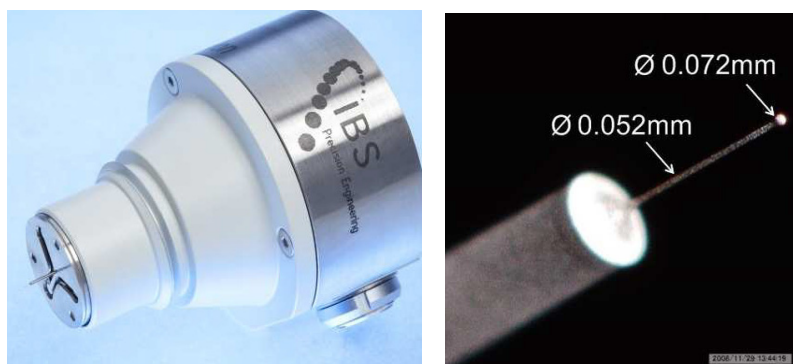

Fig. 18. IPSPE Triskelion B-500 miniature probe in CMM.

product and mirror table. The weight of the product table with work piece is directly transferred through its mounting supports to the supporting air bearings, without causing additional deformation of the zerodur mirror table.

\section{IBSPE tactile micro-probe}

The design and calibration of the newly developed "Triskelion" probe system is described in detail in reference [2]. The design features an elastically suspended stylus, which is free to deflect in $X-, Y$ - and $Z$-direction at its tip; this deflection is measured by three capacitance probes which are integrated in the probe system (Fig. 18). A miniaturized version of this probe system features a stylus with a tip diameter of about $70 \mu \mathrm{m}$. The small tip enables measurements of very small features, such as the inside diameter of very small holes (up to $1 \mathrm{~mm}$ depth). The elastic suspension of this miniaturized probe system was also redesigned to further reduce probing forces, to prevent work piece surface damage. The sensitivity calibration of this probe system is performed on an ultraprecision CMM. The probe is placed in contact with a flat work piece surface, which is located on the product table. Probe deflection is then applied by moving the table; the output signals of the probe and the interferometric table displacement are logged. Repeating this measurement for multiple probing directions yields a 3D sensitivity model. This model is validated by performing additional probing measurements. One such result is presented in Figure 19 (unfiltered data). For micro probe tip deflections less than $5 \mu \mathrm{m}$, measurement errors are less than $10 \mathrm{~nm}$ per axis of the coordinate system and less than $15 \mathrm{~nm}$ in $3 \mathrm{D}$.

The measuring uncertainty of the Isara 400 has been validated using an optical flat. The 6" optical flat was made of Zerodur and has been carefully measured optically by PTB (Physikalisch-Technische Bundesanstalt: German National Metrology Institute) using a Fizeau interferometer.

The Isara 400 was equipped with the Triskelion B500 probe. A grid of evenly distributed 4400 points has been programmed and the machine has measuring each point in touch-trigger mode (Fig. 20). The results of the Isara 400 measurement have directly been compared with the optical measurement. The comparison has been done on individual point basis, so no additional filters have been applied on the result (Fig. 21). When analysed in an his-

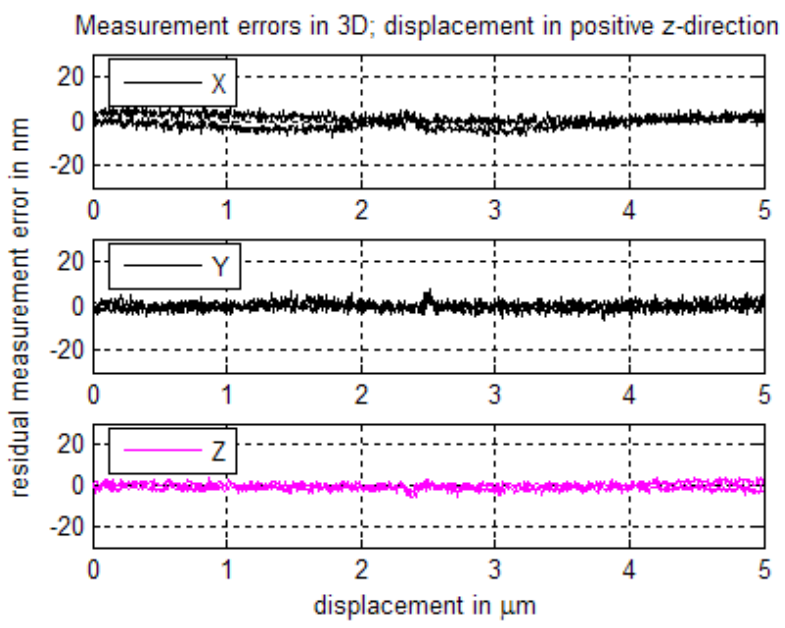

Fig. 19. Probe calibration; residual measurement errors $(x, y$, z) for probing in $z$-direction.
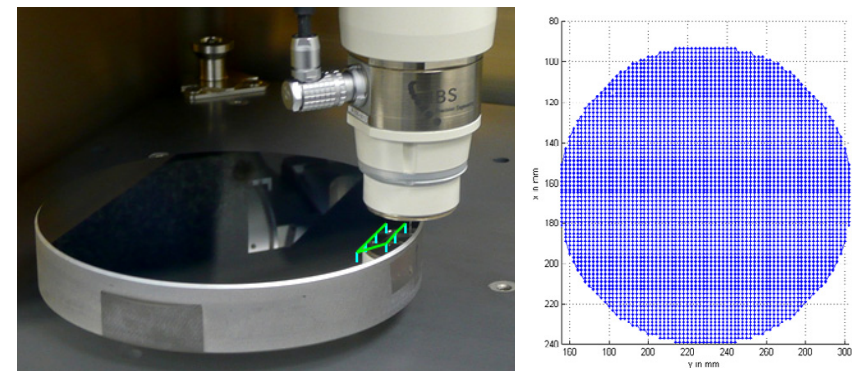

Fig. 20. IBSPE Triskelion B-500 probe (left) and distribution of the measured 4400 points (right).
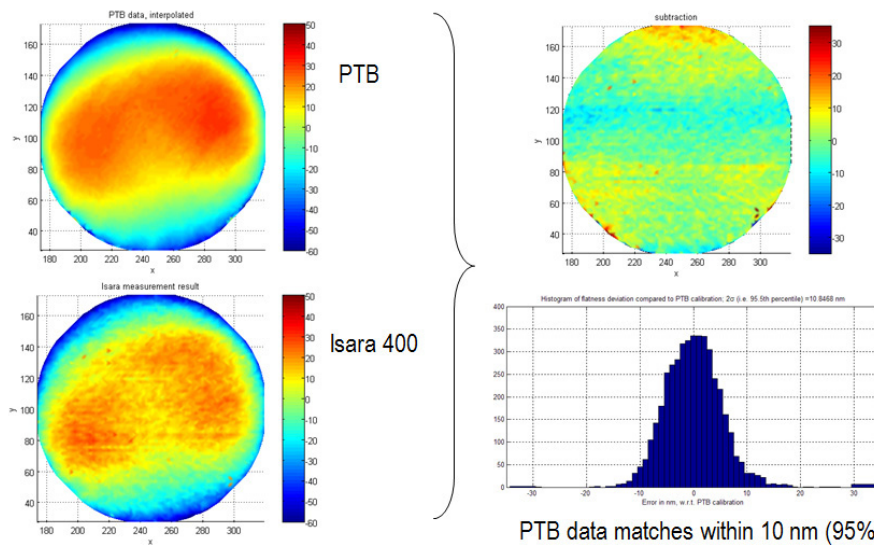

Fig. 21. Comparison of the IBSPE and PTB measurement of the flat mirror.

togram the Isara 400 matches the PTB data within $10 \mathrm{~nm}$ $(95 \%)$.

\section{Conclusion}

This paper presented existing ultra-high precision machines which are used for the calibration of the form of optical aspheric lenses with few nanometre level of accuracy. The machines designs of the LNE, VSL, SMD, 
METAS, TNO and IBSPE organizations involved together in the IND10 European EMRP project titled "Optical and tactile metrology for absolute form characterisation" were detailed. All these machines apply similar schemes such as the Abbe principles and the dissociated metrological structure principles.

These machines are equipped with tactile or optical single scanning probes with sub-nanometre resolution. Since their probing errors can be characterized separately from the machine geometric errors, theirs behaviours were investigated and relevant results were presented. The obtained results revealed that the probes do not present similar behaviours, which are usually repeatable. Such information was used to compensate the machine's errors and improving theirs measuring capabilities.

Several measurements of optical aspheric lenses were performed by the partners using tactile and optical single scanning probing systems. The results revealed the capabilities of these ultra-high precision machines to measure such kind of artefacts. A brief measurement comparison of a $500 \mathrm{~mm}$ diameter optical flat between the tactile system from IBSPE and optical systems from PTB was presented. The results revealed a similar form error of the flat within a $10 \mathrm{~nm}$ accuracy.

Acknowledgements. (1) The authors sincerely thanks the EMRP organization. The EMRP is jointly funded by the EMRP participating countries within EURAMET and the European Union (IND10). (2) The authors sincerely thanks Dr. Michael SCHULZ, coordinator of the IND10 project tilted "Optical and tactile metrology for absolute form characterization".

\section{References}

1. P. Schellekens, N. Rosielle, H. Vermeulen, M. Vermeulen, S. Wetzels, W. Pril, Design for precision: current status and trends, Ann. CIRP 47, 557-586 (1998)

2. T.A.M. Ruijl, Ultra Precision Coordinate Measuring Machine - Design, Calibration and Error Compensation, Ph.D. Thesis, Technische Universiteit Delft, 2001, ISBN 90-6464-287-7

3. K. Becker, E. Heynacher, M400 - A coordinate measuring machine with $10 \mathrm{~nm}$ resolution, Proc. SPIE 802, 209-216 (1987)

4. A. Weckenmann, G. Peggs, J. Hoffmann, Probing systems for dimensional micro- and nano-metrology, Meas. Sci. Technol. 17, 504-509 (2006)

5. I. Widdershoven, R.L. Donker, H.A.M. Spaan, Realization and calibration of the "Isara 400" ultra-precision CMM, J. Phys.: Conf. Ser. 311, 012002 (2011)

6. E. Abbe, Meßapparate für Physiker, Zeitschrift Fur Instrumentenkunde 10, 446-448 (1890)
7. A. Vissiere, H. Nouira, M. Damak, O. Gibaru, J.M. David, Concept and architecture of a new apparatus for cylindrical form measurement with a nanometre level of accuracy, Meas. Sci. Technol. 23, 094014 (2012)

8. H. Nouira, J.A. Salgado, N. El-Hayek, S. Ducourtieux, A. Delvallée, N. Anwer, Setup of a high-precision profilometer and comparison of tactile and optical measurements of standards, Meas. Sci. Technol. 25, 12 (2014)

9. H. Nouira, N. El-Hayek, X. Yuan, N. Anwer, Characterization of the main error sources of chromatic confocal probes for dimensional measurement, Meas. Sci. Technol. 25, 14 (2014)

10. R. Leach, Optical Measurement of Surface Topography (Handbook, Spring, 2011)

11. M. Vermeulen, High-Precision 3D-Coordinate Measuring Machine; Design and Prototype-Development, Ph.D. thesis, Technical University of Eindhoven, 1999

12. M.G.A. Van Veghel, R.H. Bergmans, H.J. Nieuwenkamp, Traceability of a linescale based micro-CMM, Proceedings of the Euspen international conference 2008 (Zurich, Switzerland, 2008), pp. 18-22

13. A.I. Mares, R.H. Bergmans, Evaluation of an optical coordinate measuring machine for measuring grated structures, Meas. Sci. Technol. 23, 094012 (2012)

14. Internal report VSL "Probing behavior of the VSL F25 microCMM", 2013

15. R.H. Bergmans, H.J. Nieuwenkamp, M.G.A. Van Veghel, Probing behaviour of a microCMM, Proceedings of the EUSPEN International conference (Como, Italy, 2012)

16. A. Küng, F. Meli, R. Thalmann, Ultraprecision microCMM using a low force $3 \mathrm{D}$ touch probe, Meas. Sci. Technol. 18, 319-327 (2007)

17. A. Küng, F. Meli, Versatile probes for the METAS 3D Micro-CMM, 8th Euspen Int. Conf., Zürich, Switzerland (2008), Vol. 2, pp. 338-342

18. A. Küng, F. Meli, Self calibration method for 3D roughness of spheres using an ultraprecision coordinate measuring machine, Proc. 5th Euspen Int. Conf., Montpellier, France (2005), Vol. 1, pp. 193-196

19. F. Meli, A. Küng, Scanning performances with an ultraprecision $\mu$-CMM, Proc. 6th Euspen Int. Conf., Baden bei Wien, Austria (2006), Vol. 1, pp. 193-196

20. R. Henselmans, L.A. Cacace, G.F.Y. Kramer, P.C.J.N. Rosielle, M. Steinbuch, The NANOMEFOS non-contact measurement machine for freeform optics, Precis. Eng. 35, 607-624 (2011)

21. R. Henselmans, Non-contact Measurement Machine for Freeform Optics, Ph.D. Thesis, Eindhoven University of Technology, 2009

22. L.A. Cacace, Development of a nanometre accuracy noncontact probe for NANOMEFOS, MSc Thesis, Technische Universiteit Eindhoven, nr. DCT 2006.032, 2006

23. R.L. Donker, I. Widdershoven, D. Brouns, H.A.M. Spaan, Realization of Isara 400: a large measurement volume ultra-precision CMM, Proc. ASPE annual meeting (2009) 